\title{
A Cathodoluminescence (and Raman) Imaging and Spectroscopic Study of Ancient Polycrystalline Diamond
}

\author{
E.P. Vicenzi, ${ }^{*}$ T. Rose, ${ }^{*}$ M. Fries, ${ }^{* *}$, A. Steele** and C. Magee***
}

* Department of Mineral Sciences, Smithsonian Institution, Washington, DC 20560-0119

** Geophysical Laboratory, Carnegie Institution of Washington, DC 20015

*** Research School of Earth Sciences, The Australia National University, Canberra,ACT, Australia

Determining the origin of CL spectral characteristics in many minerals is a difficult to intractable exercise owing to compositional complexities (effects of solid solution, trace and ultra-trace impurities) and/or the deformation history of the specimen. Fortunately diamonds consist of a single major chemical component, carbon, and thus represent a significantly less complicated material to interpret CL imaging and spectroscopic data. We have chosen to examine ancient polycrystalline diamond ( $>2$ billion yrs old) from Brazil for this study because of its rare microstructure, namely, shared CL features characteristic of single crystal diamond and a polycrystalline variety known as carbonado. Single crystal diamond is familiar to the general public because of its large perceived value in the jewelry market. Conversely, carbonado is a virtually unknown variety of natural polycrystalline diamond whose origin remains enigmatic as the pea- to fist-sized nodules they form have never been found in direct association with their parent rock type $[1,2]$.

The most common single crystal diamond CL characteristic is strong emission in the blue. This spectral feature has been attributed to extrinsic luminescence and the presence of nitrogen impurities [3]. Carbonados have suffered a strange coexistence with uranium early in their history, and the ensuing radiation damage from $\mathrm{MeV}$ alpha scattering and recoil has created optical centers that luminesce at frequencies significantly different from centers not associated with radiation [4, and references therein]. Specimen L5 from Lencoise in the northeastern state of Bahia, Brazil exhibits characteristics of both such CL domains (Fig 1). It appears that the inherit diamond CL signature (blue) forms the original texture of the specimen (Fig 2A). Longer wavelength features are highly correlated with cracks, apparently ancient in origin, that suggest they were an infiltration pathway for uranium. Further evidence for radiation damage overprinting can be found in seemingly isolated circular radiation halos (Figs $1 \mathrm{~B}$ and $2 \mathrm{C}$ ). Where radiation damage is particularly intense CL emission is significantly muted owing to local disruption (and amorphization) of the diamond lattice (Fig. 3 spectrum 3) [4]. CL imagery in this specimen reveals both chemical impurity and structural information. To compliment CL data obtained from both the luminoscope and SEM-based instrumentation (PMT serial spectra and CCD array detector parallel spectra), we have also performed hyperspectral Raman imaging. Of note is the striking correlation between the $1420 \mathrm{~cm}^{-1}$ band, a measure of the defect band intensity, and the long wavelength features in the visible CL.

References

[1] S. Haggerty et al., Science 285 (1999) 851-860.

[2] P.J. Heaney et al, Elements. 1 (2005) 85-89.

[3] Yacobi, B.G. and Holt, D.B. (1990) Cathodoluminescence Microscopy of Inorganic Solids: New York, Plenum, 292

[4] C. Magee (2001) Geologic, Microstructural, and Spectroscopic Constraints on the Origin and History of Carbonado Diamond. Ph.D., The Australian National University. 

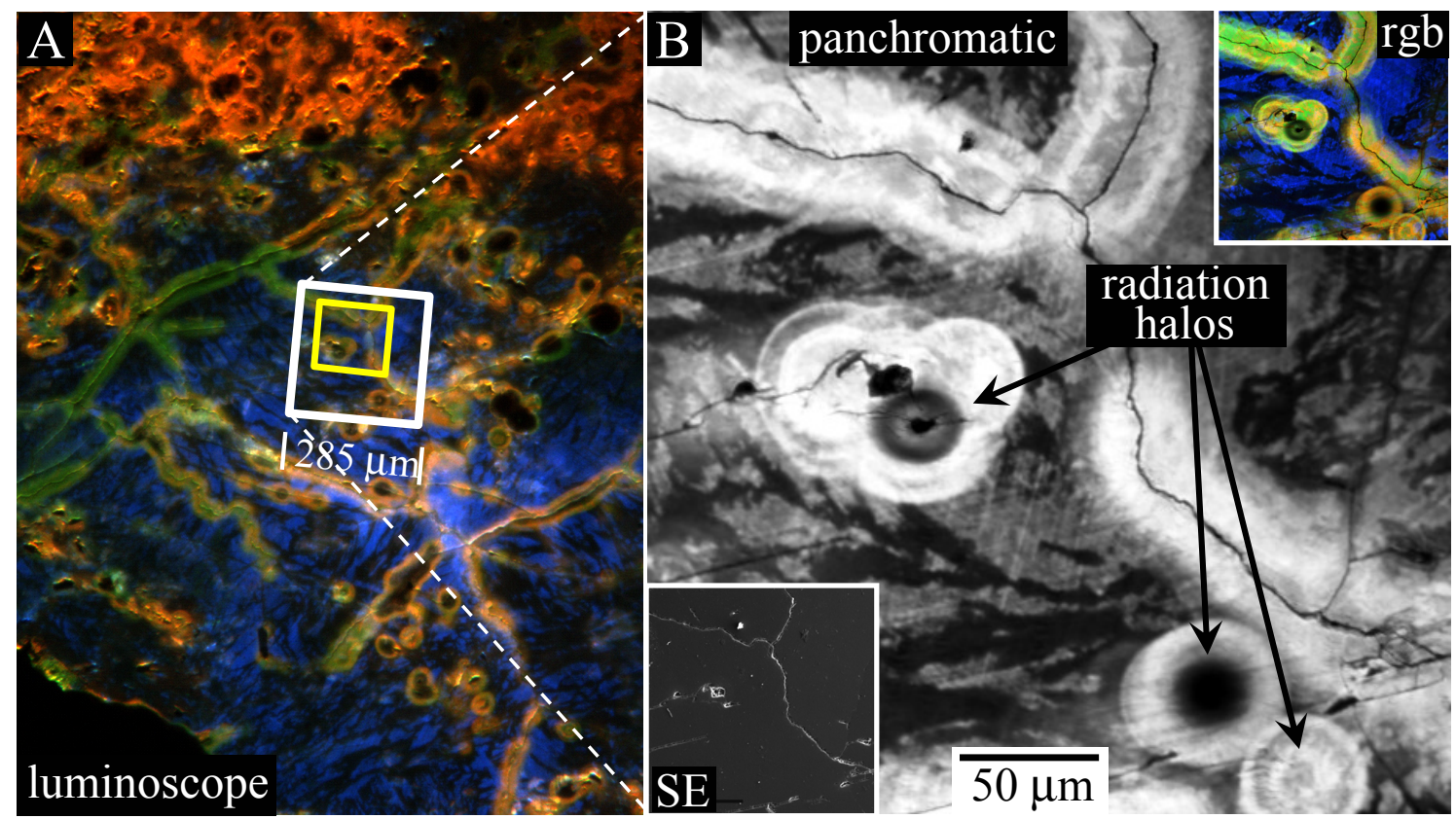

FIG. 1. A) luminoscope (CCD) overview CL image of Brazilian carbonado diamond. B) panchromatic (PMT) CL image of larger (white) rectangle in A; lower left insetsecondary electron image; upper right inset- RGB composite image.

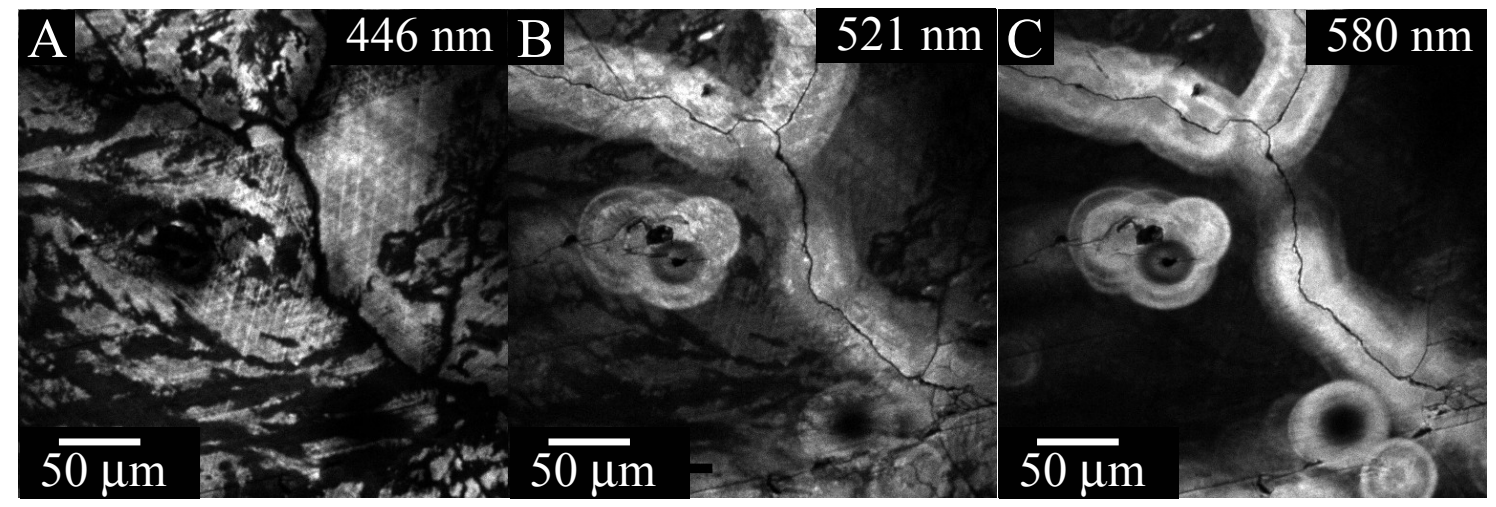

FIG. 2. Monochromatic (PMT) CL images, same area in figure $1 \mathrm{~B}$, for major features noted in the composite serial spectrum. A) $446 \mathrm{~nm}$. B) $521 \mathrm{~nm}$. C) $580 \mathrm{~nm}$
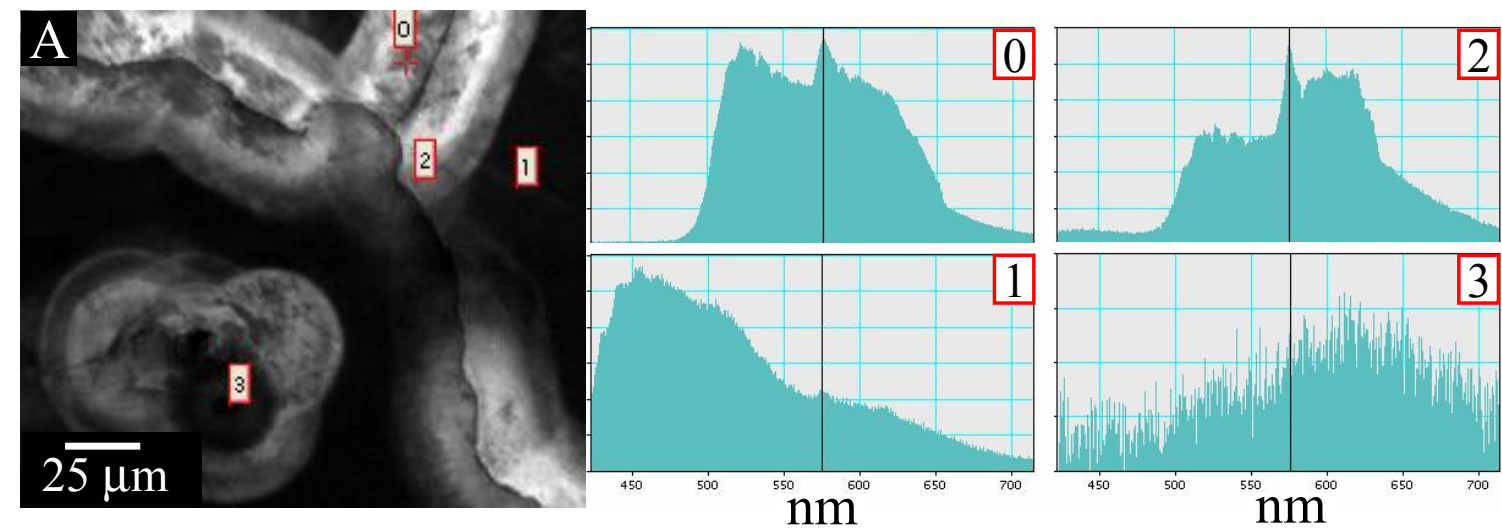

FIG. 3. Hyperspectral CL data from smaller (yellow) rectangle in 1A. A) the $699.5 \mathrm{~nm}$ image slice $(0.2 \mathrm{~nm}$ in width) with 4 pixel locations marked 0 through 3 showing dramatically different diamond spectral characteristics within the visible range (vertical line at $575 \mathrm{~nm}$ ). 